\title{
Path-integral approximation on the stability of large bipolarons in quasi-one-dimensional confinement
}

\author{
R. T. Senger and A. Erçelebi \\ Department of Physics, Bilkent University, 06533 Ankara, Turkey
}

(Received 6 July 1999)

\begin{abstract}
The stability of the singlet optical bipolaron is investigated in quasi-one-dimensional confinement with parabolic boundary potential. Under the bulk-phonon approximation the Feynman-polaron model is used to display the polaron-bipolaron phase diagram as a function of the Coulomb and phonon coupling strengths and the degree of confinement.
\end{abstract}

\section{INTRODUCTION AND THEORY}

It was conjectured by Vinetskii and Gitterman, ${ }^{1}$ and subsequently by many other scientists, that bipolarons can be realized in selective materials where a pair of two electrons can form a composite bound state in which the phononmediated attraction holds the particles together against their Coulomb repulsion. As one of the pioneering works in this area we should mention the paper by Bishop and Overhauser $^{2}$ where they explore the phonon-mediated interaction between two electrons and show that for ionic crystals, the effective electron-electron potential may lead to an attractive deep potential well with a minimum occurring for particle separations as small as a few tens of Angstrom units. Over the last decade, proceeding the discovery of high- $T_{c}$ superconductivity, there has appeared a revived and extensive interest in this problem, devoted to the study of the stability criteria of bipolarons adopting different alternative models and approximating theories. Among the numerous amount of papers published within the context of twopolaron systems, we cite a few examples ${ }^{3-15}$ which are relevant to the long-range Fröhlich interaction with the optical phonons. The fundamental conclusion led by these studies is that the domain of stability of bipolarons is determined critically by the upper and lower bounds for the repulsive Coulomb and the attractive electron-phonon coupling strengths, respectively, and that a bipolaron phase can exist only at extreme strong phonon coupling. In parallel with the study of optical bipolarons, there has also appeared some alternative attention ${ }^{16,17}$ devoted to the investigation of the two polaron complex where the electrons are assumed to couple to the longitudinal acoustic phonons through the deformation potential interaction. The essential distinction which sets the acoustic bipolaron problem apart from the optical case is that in the acoustical problem the cutoff wave vector takes part in the description of the phase diagram as a further parameter in addition to the Coulomb and phonon coupling parameters; thus leading to a much richer and interesting content of the phase picture in comparison with the equivalent case of optical bipolarons. For an extensive understanding of the salient features encountered for acoustic bipolarons the reader is referred to the two articles by da Costa and Peeters ${ }^{16}$ and by Farias, da Costa, and Peeters ${ }^{17}$ where they provide an elaborate overview to the problem in both three and two dimensions. In our present article we shall disregard any pertinence to acoustic phonon coupling and give all emphasis to the optical bipolaron problem.

Of particular relevance to our main concern in this work is the recent literature ${ }^{5-7,11,15}$ pertaining to the study of optical bipolarons in two space dimensions (2D) where it has been noted that bipolaron formation should be more favorably attained due to the pronounced phonon coupling in lowering the dimensionality from 3 to 2 . We are thus tempted to retrieve the same problem in a configuration of even lower dimensionality, namely in a quasi-one-dimensional (Q1D) wirelike geometry, where the polaron binding can be stronger and even much deeper than in two dimensions. ${ }^{18}$ In our model we assume a rather simple situation consisting of the two-polaron complex in a "free-stand" tubular configuration where the pair of electrons are free along one axis, but laterally confined in the remaining directions. The composite system is then thought of as immersed in a bosonic reservoir where they couple to the LO branch of the bulk phonon spectrum. We should emphasize that the fundamental approach followed in this work is to take into account solely the generic low dimensional aspect of the dynamical behavior of the confined electrons and visualize them as interacting with the medium and with one another through exchange of virtual LO phonons. We retain the problem in a simple form and refrain from including any modifications such as those due to the contributions from all of the other kinds of phonon modes, the screening effects and further other detailed features. Thus adopting the so-called bulk phonon approximation we wish to shed some insight into the possibility of a bipolaron phase in quasi-one-dimensional media. In the following we shall concern ourselves with the intrinsic effect of the degree of confinement on the phonon-mediated interaction acting between the particles and investigate the polaronbipolaron transition as a function of the effective dimensionality encompassing the 3D and Q1D configurations. We believe, the methodology followed in this work proves to be a powerful technique intended to lay out a satisfactory characterization of the problem in the overall ranges of the parameters describing the system. We treat our model within a similar framework of the three- and two-dimensional bipolaron models set up earlier by Verbist, Peeters, and Devreese $^{5}$ where they reformulate the Feynman "onepolaron" path-integral variational theory ${ }^{20}$ to treat the case of two interacting polarons. 


\section{A. Hamiltonian}

Setting $\hbar \omega_{\mathrm{LO}}$ as a unit of energy and $\left(\hbar / m^{*} \omega_{\mathrm{LO}}\right)^{1 / 2}$ as a unit of length, the relevant Hamiltonian reads as

$$
\begin{aligned}
\mathrm{H}= & \sum_{i=1,2}\left(\frac{1}{2} \vec{p}_{i}^{2}+\frac{1}{2} \Omega^{2} \varrho_{i}^{2}\right)+\frac{U}{\left|\vec{r}_{1}-\vec{r}_{2}\right|}+\sum_{\vec{Q}} a_{\vec{Q}}^{\dagger} a_{\vec{Q}} \\
& +\sum_{i=1,2} \sum_{\vec{Q}} V_{Q}\left(a_{\vec{Q}} e^{i \vec{Q} \cdot \vec{r}_{i}}+a_{\vec{Q}}^{\dagger} e^{-i \vec{Q} \cdot \vec{r}_{i}}\right)
\end{aligned}
$$

in which $a_{\vec{Q}}$ and $a_{\vec{Q}}^{\dagger}$ denote the phonon operators and $\vec{r}_{i}$ $=\left(\vec{\varrho}_{i}, z_{i}\right)(i=1,2)$ are the electron positions in cylindrical coordinates. Similarly, $\vec{p}_{i}(i=1,2)$ denote the respective momenta of the electrons. The Fröhlich interaction amplitude is related to the phonon wave vector $\vec{Q}=\left(\vec{q}, q_{z}\right)$ through $V_{Q}$ $=(2 \sqrt{2} \pi \alpha)^{1 / 2}|\vec{Q}|^{-1}$, where $\alpha=\left(e^{2} / \sqrt{2}\right)\left(\epsilon_{\infty}^{-1}-\epsilon_{0}^{-1}\right)$ is the coupling constant. In the Coulomb term, the unscreened amplitude $U$ is related to the ratio of the dielectric constants, $\eta=\epsilon_{\infty} / \epsilon_{0}$, through $U=\sqrt{2} \alpha /(1-\eta)$.

The dimensionless frequency $\Omega$ will be treated as a tunable parameter to serve as a measure of the degree of confinement of the electrons. The usage of a harmonic-oscillator potential greatly facilitates the calculations and leads to concise and tractable analytic expressions due to its compatibility with the framework of the path-integral approximation where one assumes the two electrons to be coupled to one another and to the corresponding fictitious particles via harmonic interactions. Moreover, due to the absence of an abrupt variation in the medium properties, the parabolic confining potential allows one to omit any interaction with the interface phonon modes. ${ }^{21}$

A complementary remark regarding the particular parabolic form of the potential used here is that it also finds its relevance in the study of bipolarons under external magnetic fields, where a field for a 3D bipolaron conforms it effectively to a quasi-one-dimensional bipolaron and a field applied normally to a quasi-two-dimensional system makes it effectively zero dimensional. Specifically, under the symmetric gauge for the magnetic vector potential, one readily has the cyclotron frequency to undertake the role of the confining parameter $\Omega$. Indeed, it has been verified recently that a magnetobipolaron in 3D behaves like a 1D bipolaron with a renormalized phonon coupling strength, and that the two problems have a one-to-one correspondence in the limit of a strong magnetic field. ${ }^{22}$

\section{B. Trial action}

In the Feynman path-integral representation of the polaron, the phonon variables can be projected out exactly to yield the partition function of the bipolaron system in the product form

$$
\begin{aligned}
\mathcal{Z}= & \prod_{\vec{Q}}\left(1-e^{-\beta}\right)^{-3} \\
& \times \prod_{i=1,2}\left(\int d \vec{r}_{0} \int_{\vec{r}_{i}(0)=\vec{r}_{0}}^{\vec{r}_{i}(\beta)=\vec{r}_{0}} \mathcal{D} \vec{r}_{i}(\lambda)\right) e^{\mathcal{S}\left[\vec{r}_{1}(\lambda), \vec{r}_{2}(\lambda)\right]}
\end{aligned}
$$

in which the action $\mathcal{S}$, expressed in imaginary time ( $t$ $\rightarrow-i \lambda)$, has the form

$$
\mathcal{S}=-\int_{0}^{\beta} d \lambda \sum_{i=1,2} \frac{1}{2}\left[\vec{r}_{i}^{2}(\lambda)+\Omega^{2} \varrho_{i}^{2}(\lambda)\right]+\mathcal{S}_{\mathrm{C}}+\mathcal{S}_{\mathrm{e}-\mathrm{ph}},
$$

where

$$
\mathcal{S}_{\mathrm{C}}=-\int_{0}^{\beta} \frac{U d \lambda}{\left|\vec{r}_{1}(\lambda)-\vec{r}_{2}(\lambda)\right|}
$$

is the Coulomb term, and $\mathcal{S}_{\text {e-ph }}$ is the phonon mediated retarded attractive interaction between the electrons, given by

$$
\begin{aligned}
\mathcal{S}_{\mathrm{e}-\mathrm{ph}}= & \frac{1}{2} \sum_{i, j=1,2} \sum_{\vec{Q}} V_{Q}^{2} \int_{0}^{\beta} d \lambda \\
& \times \int_{0}^{\beta} d \lambda^{\prime} G_{1}\left(\lambda-\lambda^{\prime}\right) e^{i \vec{Q} \cdot\left[\vec{r}_{i}(\lambda)-\vec{r}_{j}\left(\lambda^{\prime}\right)\right]} .
\end{aligned}
$$

In the above, $\beta=1 / k T$, and the memory function

$$
G_{\omega}(u)=\cosh |u| \omega\left(\operatorname{coth} \frac{1}{2} \beta \omega+\tanh |u| \omega\right)
$$

is the harmonic oscillator Green's function with frequency $\omega$.

Due to the analytic complexity comprised by the action $\mathcal{S}$, the exact ground-state energy, $E_{\mathrm{g}}=-\lim _{\beta \rightarrow \infty} \beta^{-1} \log \mathcal{Z}$, cannot be determined. Nevertheless, it is always possible to obtain a convenient variational upper bound to $E_{\mathrm{g}}$, led by the Jensen-Feynman inequality

$$
E_{\mathrm{g}} \leqslant E_{0}-\lim _{\beta \rightarrow \infty} \frac{1}{\beta}\left\langle\mathcal{S}-\mathcal{S}_{0}\right\rangle_{\mathcal{S}_{0}}
$$

where $\mathcal{S}_{0}$ refers to a solvable approximate trial action with corresponding ground-state energy $E_{0}$. The notation \langle\rangle$_{\mathcal{S}_{0}}$ denotes a path-integral average with density function $e^{\mathcal{S}_{0}}$.

For the trial action we adopt the model which has already been applied to similar (bi)polaron problems ${ }^{5,15,18-20}$ where the electrons are considered to be in quadratic interaction with the fictitious masses. Through a set of four variational parameters, $\left\{w, \omega_{1}, \omega_{2}, \omega_{3}\right\}$, we approximate the Coulomb and electron-phonon interactions harmonically as

$$
\mathcal{S}_{\mathrm{C}}=\frac{1}{4}\left(\omega_{1}^{2}-\omega_{2}^{2}-\omega_{3}^{2}\right) \int_{0}^{\beta} d \lambda\left[\vec{r}_{1}(\lambda)-\vec{r}_{2}(\lambda)\right]^{2}
$$

$$
\begin{aligned}
\mathcal{S}_{\mathrm{e}-\mathrm{ph}}= & -\frac{1}{2} \int_{0}^{\beta} d \lambda \int_{0}^{\beta} d \lambda^{\prime} G_{\mathrm{w}}\left(\lambda-\lambda^{\prime}\right)\left\{c _ { \mathrm { s } } \sum _ { i = 1 , 2 } \left[\vec{r}_{i}(\lambda)\right.\right. \\
& \left.\left.-\vec{r}_{i}\left(\lambda^{\prime}\right)\right]^{2}+2 c_{\mathrm{m}}\left[\vec{r}_{1}(\lambda)-\vec{r}_{2}\left(\lambda^{\prime}\right)\right]^{2}\right\},
\end{aligned}
$$


where the coefficients $c_{\mathrm{s}}$ and $c_{\mathrm{m}}$ refer to the self- and mutual interaction of the electrons with the fictitious masses, each with its own and with that of the remaining electron, respectively, and are given by

$$
\left\{\begin{array}{l}
c_{\mathrm{s}} \\
c_{\mathrm{m}}
\end{array}\right\}=\frac{1}{8 w}\left\{w^{2}\left(\omega_{1}^{2}-w^{2}\right) \pm\left(\omega_{2}^{2}-w^{2}\right)\left(w^{2}-\omega_{3}^{2}\right)\right\} .
$$

\section{Ground-state energy}

Since the trial action $\mathcal{S}_{0}$ and the relevant path integrals are all separable in the Cartesian coordinates, the calculations can be performed in identical manners for each spatial direction. Diagonalization of the part of the associated Lagrangian relevant to any chosen coordinate brings about four normal modes of oscillation along that coordinate with eigenfrequencies

$$
\begin{aligned}
\left\{\begin{array}{c}
\xi_{0}(\Omega) \\
\xi_{1}(\Omega)
\end{array}\right\}= & \frac{1}{\sqrt{2}}\left\{\Omega^{2}+\omega_{1}^{2} \mp \sqrt{\left(\Omega^{2}+\omega_{1}^{2}\right)^{2}-4 \Omega^{2} w^{2}}\right\}^{1 / 2}, \\
\left\{\begin{array}{c}
\xi_{2}(\Omega) \\
\xi_{3}(\Omega)
\end{array}\right\}= & \frac{1}{\sqrt{2}}\left\{\Omega^{2}+\omega_{2}^{2}+\omega_{3}^{2}\right. \\
& \left. \pm \sqrt{\left(\Omega^{2}+\omega_{2}^{2}+\omega_{3}^{2}\right)^{2}-4\left(\Omega^{2} w^{2}+\omega_{2}^{2} \omega_{3}^{2}\right)}\right\}^{1 / 2} .
\end{aligned}
$$

Here, it should be understood that the confining parameter $\Omega$ is relevant only to the transverse coordinates, and it has to be accounted for having zero value along the wire axis. Clearly, in the longitudinal $\pm z$ directions the eigenfrequencies reduce to $\xi_{0}(0)=0$ and $\xi_{i}(0)=\omega_{i}(i=1,2,3)$, in which $\omega_{1}, \omega_{2}$, and $\omega_{3}$ are the characteristic frequencies calculated previously for the bulk case by Verbist, Peeters, and Devreese. ${ }^{5}$

The normal-mode frequencies $\xi_{i}$ given by Eqs. (10) and (11) enable us to express the zero-point energy $E_{0}$ (corresponding to $\mathcal{S}_{0}$ ) as

$$
E_{0}=-3 w+\frac{1}{2} \sum_{i=0}^{3}\left\{\xi_{i}(0)+2 \xi_{i}(\Omega)\right\}
$$

wherein the additional term " $-3 w$ " comes about under eliminating the fictitious mass coordinates in obtaining the trial action.

In order to reach the upper bound to the bipolaronic ground-state energy, one has to evaluate the path-integral average $\left\langle\mathcal{S}-\mathcal{S}_{0}\right\rangle_{\mathcal{S}_{0}}$ involved in the Jensen-Feynman inequality (6). Following a series of tedious but straightforward algebra we obtain the variational bipolaron energy in the form

$$
\begin{aligned}
E_{\mathrm{g}}= & -3 w+\sum_{n=1,2} \frac{1}{n} \sum_{i=0}^{3} \xi_{i}\left(\Omega_{n}\right) \\
& +\frac{U}{\sqrt{\pi D_{1,2}\left(0, \Omega_{2}\right)}} F\left(\frac{D_{1,2}\left(0, \Omega_{1}\right)}{D_{1,2}\left(0, \Omega_{2}\right)}\right) \\
& -\sum_{n=1,2} \frac{1}{2 n}\left\{\frac{\omega_{1}^{2}-w^{2}}{\xi_{0}\left(\Omega_{n}\right)+\xi_{1}\left(\Omega_{n}\right)}+\frac{\omega_{2}^{2}+\omega_{3}^{2}-w^{2}}{\xi_{2}\left(\Omega_{n}\right)+\xi_{3}\left(\Omega_{n}\right)}\right. \\
& \left.+\frac{\omega_{2}^{2} \omega_{3}^{2}}{\xi_{2}\left(\Omega_{n}\right) \xi_{3}\left(\Omega_{n}\right)\left[\xi_{2}\left(\Omega_{n}\right)+\xi_{3}\left(\Omega_{n}\right)\right]}\right\} \\
& -\alpha \sqrt{\frac{2}{\pi}} \int_{0}^{\infty} d \tau e^{-\tau}\left\{\frac{1}{\sqrt{D_{1,1}\left(\tau, \Omega_{2}\right)}} F\left(\frac{D_{1,1}\left(\tau, \Omega_{1}\right)}{D_{1,1}\left(\tau, \Omega_{2}\right)}\right)\right. \\
& \left.+\frac{1}{\sqrt{D_{1,2}\left(\tau, \Omega_{2}\right)}} F\left(\frac{D_{1,2}\left(\tau, \Omega_{1}\right)}{D_{1,2}\left(\tau, \Omega_{2}\right)}\right)\right\},
\end{aligned}
$$

where $\Omega_{1}$ and $\Omega_{2}$ have to be accounted for having values $\Omega$ and zero, respectively, and

$$
F(x)=\frac{\arctan (\sqrt{x-1})}{\sqrt{x-1}} .
$$

The auxiliary functions $D_{1, i}(\tau, \Omega)(i=1,2)$ have the forms

$$
\begin{aligned}
D_{1,1}(\tau, \Omega)= & \frac{1}{4} \sum_{i=0}^{3} \delta_{i}(\Omega)\left(1-e^{-\xi_{i}(\Omega)|\tau|}\right), \\
D_{1,2}(\tau, \Omega)= & \frac{1}{4} \sum_{i=0,1} \delta_{i}(\Omega)\left(1-e^{-\xi_{i}(\Omega)|\tau|}\right) \\
& +\frac{1}{4} \sum_{i=2,3} \delta_{i}(\Omega)\left(1+e^{-\xi_{i}(\Omega)|\tau|}\right),
\end{aligned}
$$

where

$$
\begin{aligned}
& \delta_{0}(\Omega)=\frac{1}{\xi_{0}} \frac{w^{2}-\xi_{0}^{2}}{\xi_{1}^{2}-\xi_{0}^{2}}, \quad \delta_{1}(\Omega)=\frac{1}{\xi_{1}} \frac{\xi_{1}^{2}-w^{2}}{\xi_{1}^{2}-\xi_{0}^{2}}, \\
& \delta_{2}(\Omega)=\frac{1}{\xi_{2}} \frac{\xi_{2}^{2}-w^{2}}{\xi_{2}^{2}-\xi_{3}^{2}}, \quad \delta_{3}(\Omega)=\frac{1}{\xi_{3}} \frac{w^{2}-\xi_{3}^{2}}{\xi_{2}^{2}-\xi_{3}^{2}} .
\end{aligned}
$$

\section{RESULTS}

To trace the condition for which a stable bipolaron can be realized, one has to provide a consistent comparison of the variational energy minimum of the bipolaron system with that of the single polaron case. As a reference criterion favoring the bipolaron phase we demand that the ground-state binding energy, $E_{\mathrm{b}}=2 \Omega-E_{\mathrm{g}}$, of the pair of composite polarons which presumably make up the bipolaron be greater than twice the binding energy of one single polaron, i.e., we require $E_{\mathrm{b}}-2 E_{\mathrm{b}}^{(1)}>0$ in which the one-polaron binding energy, $E_{\mathrm{b}}^{(1)}=\Omega-E_{\mathrm{g}}^{(1)}$, is to be calculated within an identical framework of the present model and formalism and under the same numerical precision. Here, we do not replay the algebraic patterns pertaining to the derivation of the one-polaron 


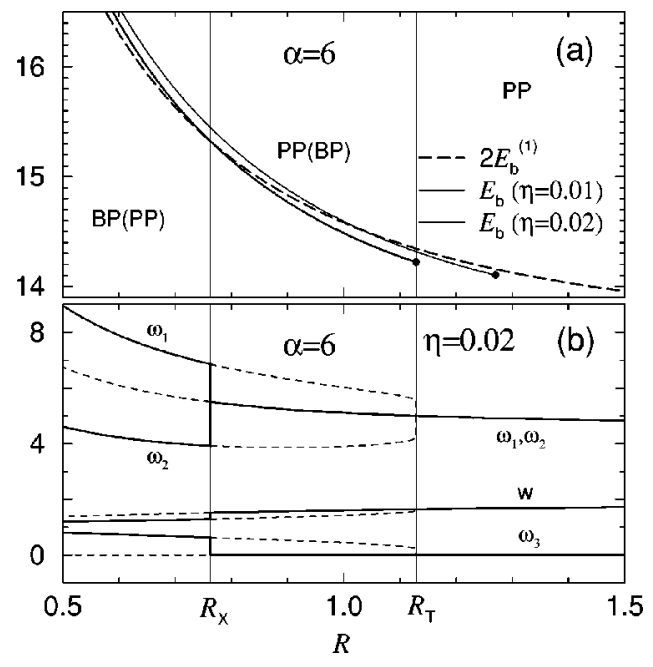

FIG. 1. (a) Bipolaron and two single-polaron binding energies, and (b) the variational parameters, as functions of $R=\Omega^{-1 / 2}$. The sample parameter values are: $\alpha=6$ and $\eta=0.01,0.02$. The crossover and cutoff radii $\left(R_{\mathrm{X}}\right.$ and $\left.R_{\mathrm{T}}\right)$ and the vertical lines separating the phases are relevant to only $\eta=0.02$.

ground-state energy, $E_{\mathrm{g}}^{(1)}$, but instead, use readily the series of Eqs. (13), (22), (25)-(30) given in a preceding paper ${ }^{18}$ concerned with the Feynman one-polaron problem consisting of the same quadratic confinement potential.

To provide an understanding of the influence of the confining potential on the evolution of the complex of two polarons in favor of a bipolaron bound state, we undertake two illustrative cases for which the Coulomb repulsion is considered to be not too strong, and the phonon mediated binding somewhat deep enough to overcome that repulsion. Setting $\alpha=6$ and $\eta=0.01,0.02$ as sample values, we display [cf. Fig. 1(a)] a graphical comparison of the corresponding binding energies in the polaron-polaron (PP) and bipolaron (BP) phases as a function of $R=\Omega^{-1 / 2}$ which we hereafter refer to as simply the radius. A glance at the plots in Fig. 1(a) reveals that there are three major regions in the overall domain of $R$. For $R<R_{\mathrm{X}}$, i.e., in the region $\mathrm{BP}(\mathrm{PP})$, the bipolaronic phase is energetically more favorable than the PP phase of two individual polarons, and yet, decreasing the wire radius enhances the stability of the BP phase. In going toward the opposite extreme and relaxing the degree of confinement, the BP state can no more be sustained, and beyond the crossover radius $R_{\mathrm{X}}$, the bipolaronic phase is seen to persist recessively as a metastable state and eventually terminate at a cutoff radius $R_{\mathrm{T}}$ (shown in the figure by a dark dot). For $R$ $\geqslant R_{\mathrm{T}}$ one achieves solely the PP phase with whatsoever no relevance to any possible bipolaronic characterization of the two-polaron system. An additional remark led by the content of the Fig. 1(a) is that, for a given fixed $\alpha$, the stronger the Coulomb repulsion is, the more is the requirement for a thinner wire to favor and sustain the bipolaron state. In this regard, for $\alpha=6$, we have calculated $R_{\mathrm{X}}=1.03(0.76)$ and $R_{\mathrm{T}}$ $=1.27(1.13)$ for $\eta=0.01(0.02)$. For completeness, we also display complementary plots of the variational parameters as a function of the transition from one phase to the other [cf. Fig. 1(b)]. The parameters pertaining to the energetically favorable phase are plotted in boldface curves and those which correspond to the metastable state of either phase are given

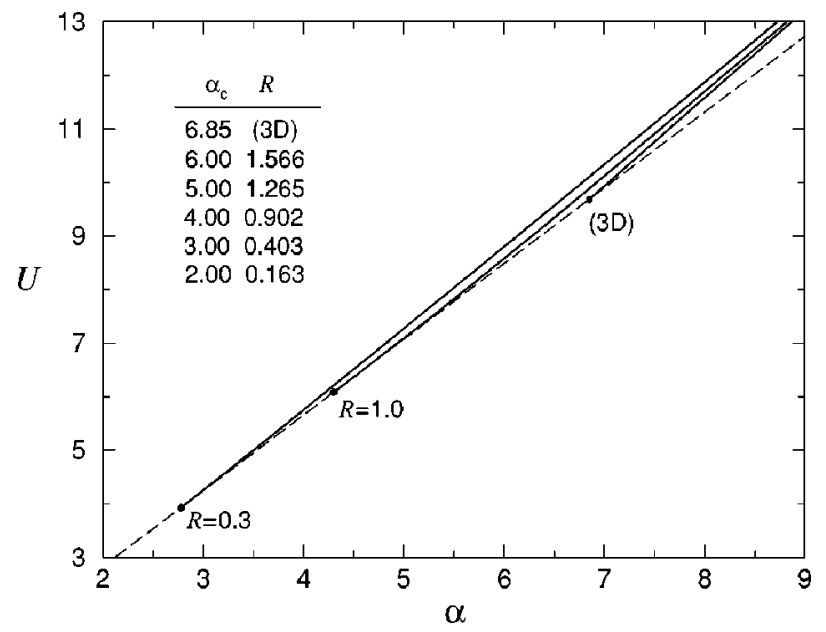

FIG. 2. The phase diagram for bipolaron formation in the space of the Coulomb coefficient and the electron-phonon coupling constant. The solid lines from top to bottom are, respectively, for $R$ $=0.3,1.0$ and $R \rightarrow \infty$. The dashed line $(\eta=0)$ is the boundary of the unphysical region. The inset tabulates the corresponding radii for a few sample values of $\alpha_{c}$.

in dashed lines. It should be noted that if the bipolaronic phase should indeed take place either as a metastable state or otherwise, the corresponding variational parameters all achieve nonzero and distinctive values. The phase of two independent polarons, however, is characterized by that $\omega_{1}$ and $\omega_{2}$ coalesce into one single curve, and $\omega_{3}=0$ regardless of $R$. Consequently, one has $w$ and $\nu\left(=\omega_{1}=\omega_{2}\right)$ to be recognized as the well established Feynman variational parameters of the one-polaron problem. ${ }^{18,20}$

In Fig. 2 we plot a phase diagram in the " $U-\alpha$ " plane, picturing the domain of stability of the bipolaron state at different degrees of confinement. In the diagram the space lying below the dashed line corresponds to $\eta<0$, and is therefore irrelevant. The upper space bounded from below by the solid lines plotted for three distinctive radii $(R=0.3, R$ $=1.0$, and $R \gg 1$ ) gives the unstable region. It is therefore only the narrow triangular area bounded by the dashed and either of the solid lines in which the polarons can be found in a stable bound state forming a bipolaron. The vertex of each triangular region at which the dashed and solid lines join defines a critical minimum for the coupling constant in the bulk and Q1D $(R=1.0$, and 0.3) configurations. Coupling constants larger than these critical vertex values serve for supporting the bipolaron to maintain its stability at correspondingly stronger Coulomb repulsions. In numerical terms, setting $\alpha=8$ for instance, we evaluate the critical upper bound for the Coulomb coefficient beyond which the bipolaron dissociates into two individual polarons as $U_{c}$ $=11.59,11.70$, and 11.88, respectively, for the cases $R$ $=$ bulk, 1.0 , and 0.3 .

A more complete trace of the critical condition on the Coulomb strength as a function of the degree of confinement is given in Fig. 3 where we provide a layout of the alternative related parameter $\eta$ against $R$ plotted for a sequence of coupling constants ranging from 4 to 10 . An immediate glance at the series of plots in Fig. 3 reveals that, starting from the bulk limit, $\eta_{c}$ displays in general an increasing trend with decreasing $R$, yielding an explicit evidence in fa- 


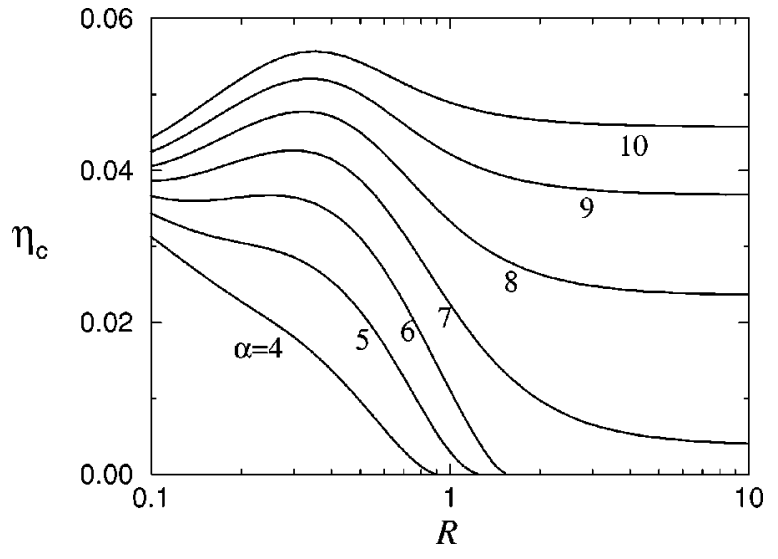

FIG. 3. The critical ratio $\eta_{c}$ as a function of $R=\Omega^{-1 / 2}$.

vor of a pronounced effective phonon coupling with the increase in the degree of confinement, which correspondingly results in an enhancement in the phonon mediated interaction between the electrons to serve for opposing and counterbalancing even more stronger Coulomb repulsions. Examining the low-lying plots in the figure, we note that for $\eta=0$, i.e., for the Coulomb coefficient taken as assuming its hypothetical minimum value $(U=\sqrt{2} \alpha$ ), the critical coupling constant over which the bipolaron phase is realized is found to be $\alpha_{c}^{(3 \mathrm{D})}=6.85$ for the bulk case. ${ }^{5}$ Correspondingly, we note that our plots of $\eta_{c}$ for $\alpha=4,5,6$ all intersect the abscissa at finite values of $R$; hence, for not strong enough $\alpha$, a bipolaron can form only beyond a critical degree of confinement; and the smaller the coupling constant is, the smaller is the corresponding critical radius. Clearly, for $\alpha>6.85$ one has $R_{c} \rightarrow \infty$, where in this case bipolaron formation is favorable even in bulk, and the additional effect of the confinement is to take over in favor of enhancing the already established stability of the bipolaron.

We would like to draw attention to that, for comparatively large $\alpha$, the critical $\eta$ exhibits a peculiar variation as the wire radius is decreased; compatible with our anticipations, it first increases, and after having gone through a maximum, displays a decreasing trend. We feel that this salient feature is an implicit consequence of the fact that the parameters $\alpha, \eta$, and $\Omega$ which characterize the system do not enter the problem in an independent way but all together take part in the binding in related manners through an entangled admixture of the competing effects of the Coulomb repulsion and the phonon mediated attraction over the other and the crossovering between these counter aspects as the confining parameter is varied. Clearly, in a confined volume where $\Omega$ is tuned from zero to large values, it is not only the phonon coupling which gets pronounced, but in the meantime the kinetic energy of the electrons increases and the Coulomb repulsion becomes steadily strengthened due to that the particles are squeezed to get closer. Thus the overall role of the confinement on the phonon-coupling induced localization of the electron-electron pair and the withstanding kinetic and Coulomb repulsions is to make these competing counter aspects stronger. What is more peculiar to the present context is that during when $\Omega$ is varied, the phonon coupling and the Coulomb strengths may not in general grow or decrease monotonically in concert at the same rate and consequently, the relative dominating strength of either the Coulomb potential

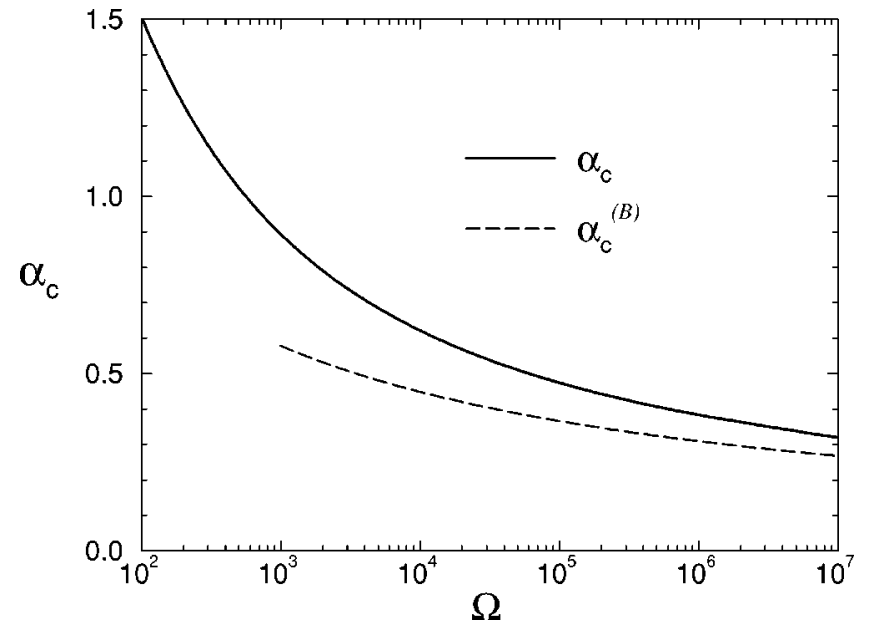

FIG. 4. The critical coupling constant $\alpha_{c}$ as a function of $\Omega$ in the extreme high confinement limit. The solid curve reflects the results of the present model. The dashed curve is the asymptotic 1D relation: $\alpha_{c}^{(B)}=4.6 / \ln B$ derived by Smondyrev et al. (Ref. 22) for the $3 \mathrm{D}$ magnetobipolaron in the strong magnetic-field limit. For compatibility, we have set $B=2 \sqrt{2} \Omega$.

or the electron-phonon interaction over the other may become altered as a function of the degree of confinement. In the particular Q1D configuration, with contracting wire radius, the electrons experience an increasingly large restriction toward the wire axis and therefore, below a certain wire size, the phonon-mediated attraction starts to lose its overwhelming strength against the rapidly increasing Coulomb and kinetic repulsions thus leading to a decreasing profile of $\eta_{c}$ as $R$ is made even smaller. This feature however is seen to diminish as $\alpha$ is tuned to smaller values. For comparatively weak $\alpha$, where the electrons are not too tightly bound by the lattice, we think that, with shrinking wire size, the charge density of the electrons acquire a spatial extent expanded to relax itself in the longitudinal $( \pm z)$ directions along the wire axis, resulting in the overall a weaker repulsion between the particles; thus enabling the phononmediated attraction to maintain its capability in supporting the bipolaron state against larger values of the Coulomb coefficient, and correspondingly, larger values of $\eta_{c}$.

In this report we have studied the possibility and criteria in achieving stable bipolarons in Q1D media where the electrons are laterally confined by a cylindrical free-stand wire with parabolic boundary potential. The overall implication led by the numerical outcomes of this work is that, the stronger the phonon coupling and/or the larger the degree of confinement is, the more favorably the bipolaron state can form and be supported against the repulsive Coulomb potential. We further conclude that it is the mutual and interrelated roles which the Coulomb and phonon coupling parameters play together with the confinement that lead to the formation (or dissociation) of a bipolaron. As a final complementary remark we would like to add the note that the confined bipolaron model adopted here reproduces almost similar qualitative features as for the alternative case of a 3D bipolaron in a magnetic field. In both cases it is seen that the common fundamental effect of either the cylindrical parabolic potential or the external magnetic field is to enlarge the region of bipolaron formation so that the critical coupling constant re- 
quired to sustain the bipolaron phase assumes smaller values as the parabolic potential or the magnetic field are made stronger. The similarity between the two problems become more closer in the high magnetic-field limit $(B \rightarrow \infty)$, since it is only then the dominant contribution to the magnetobipolaron energy comes from the ground Landau level and the essential digression originating from the angular momentum term in the vector-potential part of the Hamiltonian gets removed. It has already been shown by Smondyrev et al. ${ }^{22}$ that the 3D magnetobipolaron maps onto a 1D bipolaron for strong fields and that the effective critical coupling constant scales to smaller values with proportionality to the reciprocal

${ }^{1}$ V.L. Vinetskii and M.S. Gitterman, Zh. Éksp. Teor. Fiz. 33, 730 (1957) [Sov. Phys. JETP 6, 560 (1958)].

${ }^{2}$ M.F. Bishop and A.W. Overhauser, Phys. Rev. B 23, 3627 (1981).

${ }^{3}$ J. Adamowski, Phys. Rev. B 39, 3649 (1989).

${ }^{4}$ F. Bassani, M. Geddo, G. Iadonisi, and D. Ninno, Phys. Rev. B 43, 5296 (1991).

${ }^{5}$ G. Verbist, F.M. Peeters, and J.T. Devreese, Phys. Rev. B 43, 2712 (1991).

${ }^{6}$ S. Sil, A.K. Giri, and A. Chatterjee, Phys. Rev. B 43, 12642 (1991).

${ }^{7}$ G. Verbist, M.A. Smondyrev, F.M. Peeters, and J.T. Devreese, Phys. Rev. B 45, 5262 (1992).

${ }^{8}$ J. Adamowski and S. Bednarek, J. Phys.: Condens. Matter 4, 2845 (1992).

${ }^{9}$ P. Vansant, M.A. Smondyrev, F.M. Peeters, and J.T. Devreese, J. Phys. A 27, 7925 (1994).

${ }^{10}$ C. Qinghu, W. Kelin, and W. Shaolong, Phys. Rev. B 50, 164 (1994).

${ }^{11}$ F. Luczak, F. Brosens, and J.T. Devreese, Phys. Rev. B 52, 12 of $\ln B$. Numerical treatment of the present calculation tackled in the unrealistic extreme high confinement limit $(\Omega$ $\rightarrow \infty$ ) reproduces somewhat the same nature in an asymptotic manner, where we observe that the critical coupling constant seems to bear a linkage to the confining parameter through an analogous relation: $\alpha_{c} \simeq 1 / \ln \Omega$ (cf. Fig. 4).

\section{ACKNOWLEDGMENT}

R. T. Senger acknowledges the financial support of the Münir Birsel Foundation-TUBiTAK.

743 (1995).

${ }^{12}$ M.A. Smondyrev, J.T. Devreese, and F.M. Peeters, Phys. Rev. B 51, 15008 (1995).

${ }^{13}$ S. Sahoo, J. Phys.: Condens. Matter 7, 4457 (1995).

${ }^{14}$ S. Mukhopadhyay and A. Chatterjee, J. Phys.: Condens. Matter 8, 4017 (1996).

${ }^{15}$ R.T. Senger and A. Erçelebi, Phys. Rev. B 60, 10070 (1999).

${ }^{16}$ W.B. da Costa and F.M. Peeters, J. Phys.: Condens. Matter 8, 2173 (1996).

${ }^{17}$ G.A. Farias, W.B. da Costa, and F.M. Peeters, Phys. Rev. B 54, 12835 (1996).

${ }^{18}$ R.T. Senger and A. Erçelebi, J. Phys.: Condens. Matter 9, 5067 (1997).

${ }^{19}$ F. Brosens and J.T. Devreese, Phys. Rev. B 54, 9792 (1996).

${ }^{20}$ R.P. Feynman, Phys. Rev. 97, 660 (1955).

${ }^{21}$ S.N. Klimin, E.P. Pokatilov, and V.M. Fomin, Phys. Status Solidi B 184, 373 (1994).

${ }^{22}$ M.A. Smondyrev, E.A. Kochetov, G. Verbist, F.M. Peeters, and J.T. Devreese, Europhys. Lett. 19, 519 (1992). 